\title{
Chemotherapy with stem cell transplantation is more effective than immunotherapy in sporadic late onset nemaline myopathy with monoclonal gammopathy
}

\author{
Rouslan Kotchetkov (i] ${ }^{1} \cdot$ Anna Dyszkiewicz-Korpanty ${ }^{2,3} \cdot$ Vishal Kukreti $^{4}$
}

Received: 27 November 2017 / Revised: 5 December 2017 / Accepted: 10 December 2017 / Published online: 24 January 2018

(c) The Author(s) 2018. This article is published with open access

Sporadic late onset nemaline myopathy (SLONM) is a rare proximal myopathy with an insidious onset and slow progression. Patients present initially with a proximal limb weakness. Most commonly dyspnea, dysphagia, facial muscle weakness, and cardiomyopathy develop with disease progression. If not treated, SLONM could result in $40 \%$ mortality within the first 12 months. In approximately half of the reported cases SLONM is associated with plasma cell disorders, mostly monoclonal gammopathy of unknown significance (MGUS) and anecdotally with multiple myeloma and light chain deposition disease [1-3]. SLONM associated with a monoclonal gammopathy (SLONM + MGUS) has an aggressive course of the disease, more severe weakness, earlier development of dysphagia and dyspnea, and overall carries an unfavourable prognosis [1, 2]. Two approaches are used to treat SLONM + MGUS: (1) immunosuppressive therapy (steroids, steroid-sparing agents, intravenous immunoglobulins (IVIG), and plasmapheresis/plasma exchange) or (2) chemotherapy followed by autologous stem cell transplantation (ASCT). Due to the

Rouslan Kotchetkov

kotchetkovr@rvh.on.ca

1 Division of Hematology-Oncology, Simcoe Muskoka Regional Cancer Program, Royal Victoria Health Center, 201 Georgian Drive, Barrie, ON L4M 6M2, Canada

2 Laboratory Medicine Program, Toronto General Hospital, University Health Network, University of Toronto, 200 Elizabeth St., Toronto, ON M5G 2C4, Canada

3 Division of Medical Oncology and Hematology, Department of Medicine, Princess Margaret Cancer Center, Toronto, ON, Canada

4 Division of Medical Oncology and Hematology, Department of Medicine, Princess Margaret Cancer Centre, University Health Network, University of Toronto, 610 University Avenue, Toronto, ON M5G 2M9, Canada rare nature of the disease, the best treatment modality is unknown.

A 64-year-old male without a background history of musculoskeletal disease presented with 1 year of progressive generalized muscle weakness, difficulties with ambulating and holding his posture. He subsequently developed dysphagia and dyspnea. On exam he could walk with support only, was in a mild respiratory distress, and had marked proximal weakness in the upper and lower extremities with visible muscle atrophy. His blood work was unremarkable, except for the presence of immunoglobulin $\mathrm{G}(\mathrm{IgG})$ lambda monoclonal $(\mathrm{M})$ protein of $2.7 \mathrm{~g} / \mathrm{L}$. Bone marrow biopsy showed $5 \%$ plasma cells with no light chain restriction. Further work up for an underlying HIV or malignancy was negative. He had cardiomyopathy with global systolic dysfunction (left ventricular ejection fraction decreased to 20\%). Creatinine kinase level was normal. Muscle biopsy showed variable size skeletal muscle with scattered small basophilic angulated fibers associated with mild endomysial hemolysis. Some of the muscle fibers showed dense sarcoplasmic aggregates. Electron microscopy revealed skeletal muscles with extensive myofibrillar disarray and abundant cytoplasmic nemaline rods. No rod-like inclusions were found in nuclei. Diagnostic work up did not identify any hereditary causes. The patient was diagnosed with a SLONM + MGUS and subsequently started systemic chemotherapy with cyclophosphamide, bortezomib, dexamethasone. After six cycles of this regimen he underwent conditioning with high-dose melphalan $\left(200 \mathrm{mg} / \mathrm{m}^{2}\right)$ followed by ASCT. Clinical improvement started 6 weeks after the initiation of chemotherapy and was more pronounced a month after the ASCT. Twelve months following the ASCT the patient was able to walk over $5 \mathrm{~km}$ daily, swim, and was autonomous in daily activities. Left ventricular ejection fraction improved to $55 \%$. He has no measurable M-protein. He was last seen in a follow-up at 
38 months post-transplant and has remained in complete clinical and hematological remission.

We reviewed the literature and identified 14 SLONM + MGUS patients treated with an immune-based approach (Table 1) and 14 patients with chemotherapy + ASCT (Table 2). Overall, in both groups there was a male predominance, 1.5:1 and 3.5:1, respectively, with a median age of 49 years. All patients had a small monoclonal M-protein, and all MGUS cases were exclusively IgG, with kappa to lambda distribution 1.1:1. Among 14 patients who were treated with immune-based therapy some degree of improvement was achieved in $7(50 \%)$ patients. Three patients were reported to have a significant clinical improvement. Of those, two declined ASCT and were successfully treated with immunotherapy. In both patients improvement of neurological symptoms correlating with a resolution of monoclonal protein was reported (Table 1 [1]). One patient who received IVIG monthly over 3 years reported almost complete resolution of weakness (Table 1 [2]). In two other patients who were treated with combined immunosuppression, a moderate improvement was achieved (Table $1[3,4])$. Two other patients who received combined therapy with steroids, plasma exchange, and steroid-sparing immunosuppressants had only a mild neurological improvement (Table $1[5,6])$. Only the patient treated with a combination of prednisone and cytarabine for 2.5 years has achieved stable disease (Table 1 [7]). Six patients (42\%) treated with either prednisone and IVIG monotherapy, or prednisone and immunosuppressants progressed (Table 1 [8-11]).

In contrast, when considering our experience along with the experiences reported in the literature, the treatment with ASCT resulted in significant improvement of neurological symptoms in 14/15 (93\%) patients (Table 2). Among eight patients reported by Voermans et al. [4] seven achieved a sustainable good/moderate response, with six very good partial or complete hematological responses, and one partial response. One patient showed no clinical or hematological response and died from a progressive disease. Six cases, similarly to our case, showed a significant improvement of neurological symptoms, including a complete recovery in three patients (Table 2, [2-7]). The majority of clinical and hematological responses were long-lasting, with a followup of up to 96 months (mean 18 months, range 5-96 months). Clinical response correlated with hematological response and all patients had no measurable M-protein during the reported follow-up period.

Even though the nature of the relationship between SLONM and monoclonal gammopathy is not completely clarified, several observations suggested a direct association between the presence of M-protein and the disorder. It was proposed to classify SLONM + MGUS as a plasma cell dyscrasia with toxic M-protein, similar to amyloidosis or
POEMS (polyneuropathy, organomegaly, endocrinopathy /edema, M-protein, skin abnormalities) syndrome [4]. Tissue damage may occur via several mechanisms. In immunoglobulin light chain amyloidosis amyloid depositions produced by monoclonal plasma cells result in cellular injury, tissue damage, and organ dysfunction [5]. Direct cytotoxicity of immunoglobin light chains has also been demonstrated in cases of cardiac amyloid [6]. In POEMS syndrome, it is not the aggregation and deposition of the monoclonal antibodies in affected tissue but rather the antibody activity toward autogenous antigens along with the effects of vascular endothelial growth factor (VEGF) and possibly other humoral mediators overproduction is considered causative [12]. However, both of these entities improve when the clone of plasma cells is eradicated [7, 8]. Based on these observations Voermans and colleagues suggested that high-dose melphalan + ASCT should be the first-line therapy for SLONM + MGUS [4]. A recent review also confirmed the efficacy of ASCT for the therapy of SLONM and considered SLONM + MGUS as a treatable disease with therapy directed toward plasma cell clone, including chemotherapy and ASCT [2]. Taking into account the pathophysiology of the disease, response to chemotherapy, which correlates with M-protein values, we propose to approach SLONM + MGUS as a plasma cell dyscrasia, rather than dysimmune disease. Given the low efficacy of steroids and immunosuppressants and the rapid course of the disease we recommend the use chemotherapy and ASCT with a goal to eradicate malignant plasma cells clone, similar to other plasma cell neoplasms.

Over the past few years significant advances have become available for treating plasma cell dyscrasias. The advances of therapy in multiple myeloma suggest that novel agents, such as proteasome inhibitors and immunomodulators, might be an effective therapy of SLONM + MGUS even without consolidative ASCT. A recent report also showed an efficacy of lenalidomide + dexamethasone combination in a 54-year-old female patient with SLONM associated with multiple myeloma. In this patient clinical improvement was already reported after 3 months of therapy [3]. These new data are of a particular importance for patients with comorbidities who are not eligible for ASCT. As recently reported by Belhomme et al. [9], a patient with a severe left ventricular dysfunction possibly due to MGUS related myopathy showed a significant improvement after treatment with four cycles of cyclophosphamide, bortezomib, dexamethasone without consolidation with ASCT.

SLONM + MGUS is a rare disease and our analysis is limited by its retrospective nature and a small number of published case series. More data are needed to determine the optimal management of SLONM + MGUS. Based on our experience and the literature review we conclude that chemotherapy followed by consolidative ASCT is more 


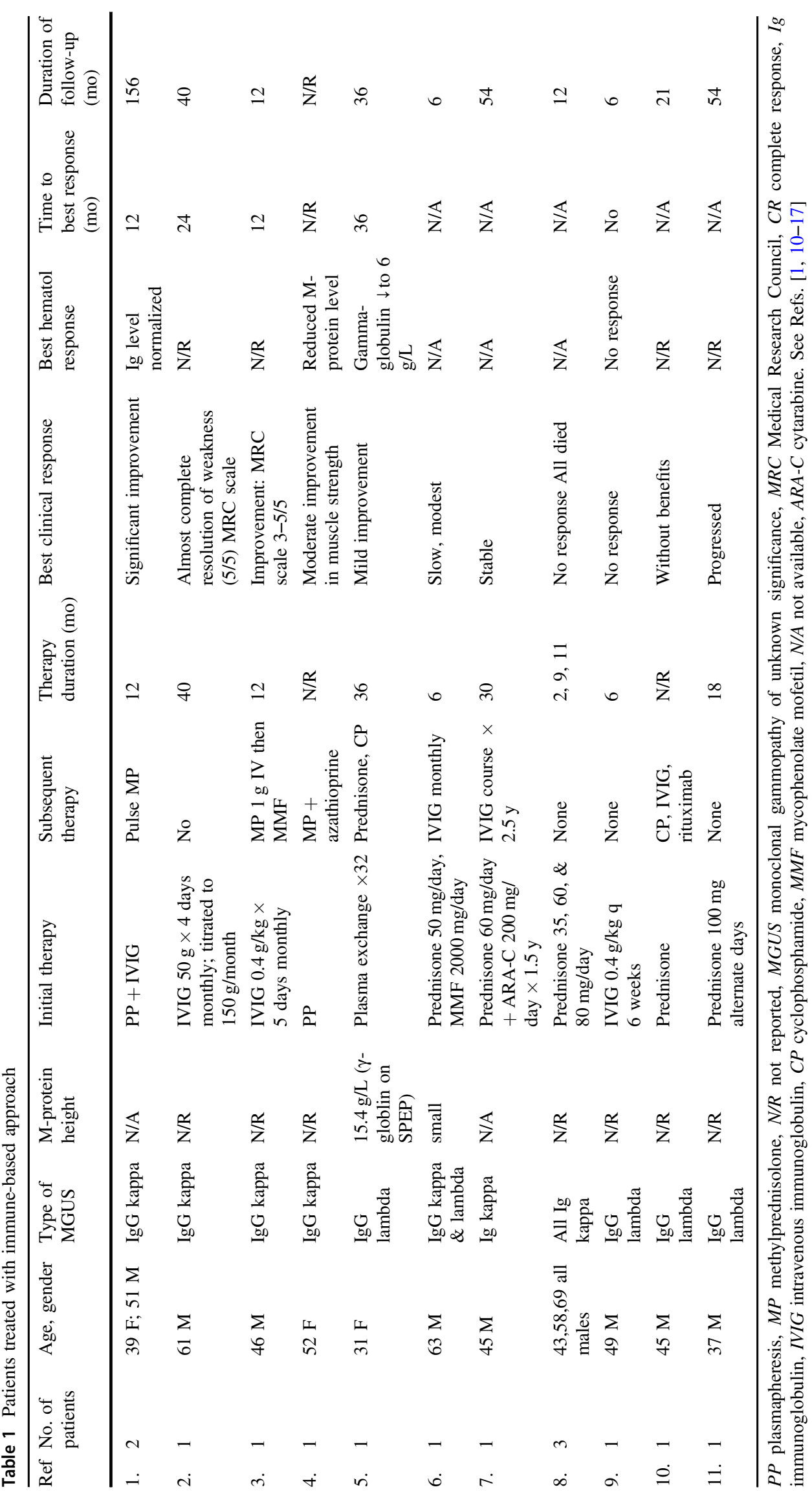




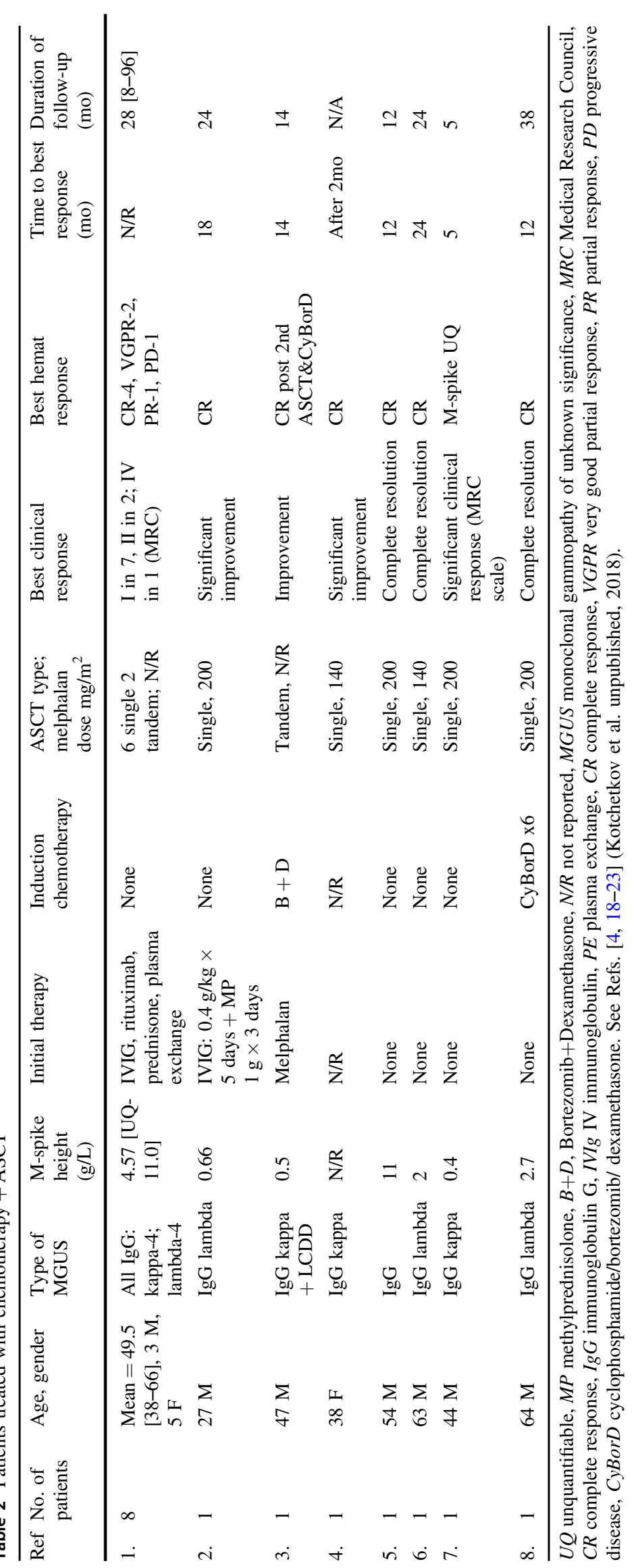


effective than immunotherapy and should be a considered as a preferred approach for patients with SLONM + MGUS.

\section{Compliance with ethical standards}

Conflict of interest The authors declare that they have no conflict of interest.

Open Access This article is licensed under a Creative Commons Attribution-NonCommercial-ShareAlike 4.0 International License, which permits any non-commercial use, sharing, adaptation, distribution and reproduction in any medium or format, as long as you give appropriate credit to the original author(s) and the source, provide a link to the Creative Commons license, and indicate if changes were made. If you remix, transform, or build upon this article or a part thereof, you must distribute your contributions under the same license as the original. The images or other third party material in this article are included in the article's Creative Commons license, unless indicated otherwise in a credit line to the material. If material is not included in the article's Creative Commons license and your intended use is not permitted by statutory regulation or exceeds the permitted use, you will need to obtain permission directly from the copyright holder. To view a copy of this license, visit http://creativecommons. org/licenses/by-nc-sa/4.0/.

\section{References}

1. Chahin N, Selcen D, Engel AG. Sporadic late onset nemaline myopathy. Neurology. 2005;65:1158-64.

2. Uruhaa A, Benveniste O. Sporadic late-onset nemaline myopathy with monoclonal gammopathy of undetermined significance. Curr Opin Neurol. 2017;30:457-63.

3. Montagnese F, Portaro S, Musumeci O, Migliorato A, Moggio M, Fagiolari G, et al. Sporadic late-onset nemaline myopathy in a woman with multiple myeloma successfully treated with lenalidomide/dexamethasone. Muscle Nerve. 2015;51:934-5.

4. Voermans NC, Benveniste O, Minnema MC, Lokhorst H, Lammens M, Meersseman W, et al. Sporadic late-onset nemaline myopathy with MGUS: long-term follow-up after melphalan and SCT. Neurology. 2014;83:2133-9.

5. Merlini G, Seldin DC, Gertz MA. Amyloidosis: pathogenesis and new therapeutic options. J Clin Oncol. 2011;29:1924-33.

6. Shi J, Guan J, Jiang B, Brenner DA, Del Monte F, Ward JE, et al. Amyloidogenic light chains induce cardiomyocyte contractile dysfunction and apoptosis via a non-canonical p38alpha MAPK pathway. Proc Natl Acad Sci USA. 2010;107:4188-93.

7. Kuwabara S, Misawa S, Kanai K, Kikkawa Y, Nishimura M, Nakaseko C, et al. Autologous peripheral blood stem cell transplantation for POEMS syndrome. Neurology. 2006;66:105-7.

8. Jaccard A, Moreau P, Leblond V, Leleu X, Benboubker L, Hermine $\mathrm{O}$, et al. High-dose melphalan versus melphalan plus dexamethasone for AL amyloidosis. New Engl J Med. 2007;357:1083-93.

9. Belhomme N, Maamar A, Le Gallou T, Minot-Myhié MC, Larralde $\mathrm{A}$, Champtiaux $\mathrm{N}$, et al. Rare myopathy associated to MGUS, causing heart failure and responding to chemotherapy. Ann Hematol. 2017;96:695-6.

10. Mizuno Y, Mori-Yoshimura M, Okamoto T, Oya Y, Nishino I, Murata M. Two cases of sporadic late onset nemaline myopathy effectively treated with immunotherapy. Rinsho Shinkeigaku. 2016;56:605-11.

11. Milone M, Katz A, Amato AA, Soderland CA, Segarceanu M, Young NP, et al. Sporadic late onset nemaline myopathy responsive to IVIG and immunotherapy. Muscle Nerve. 2010;41:272-6.

12. Deconinck N, Laterre EC, Van den Bergh PY. Adult-onset nemaline myopathy and monoclonal gammopathy: a case report. Acta Neurol Belg. 2000;100:34-40.

13. Eymard B, Brouet JC, Collin H, Chevallay M, Bussel A, Fardeau M. Late-onset rod myopathy associated with monoclonal gammopathy. Neuromuscul Disord. 1993;3:557-60.

14. Irodenko V, Lee HS, De Aarmond SJ, Layzer RB. Adult nemaline myopathy with trabecular muscle fibers. Muscle Nerve. 2009;39:871-5.

15. Hanisch F, Schneider I, Müller T, Romeike BF, Stoltenburg G, Holzhausen HJ, et al. Behandelbarkeit der, sporadic late onset nemaline myopathy“. Nervenarzt. 2013;84:955-61.

16. Keller CE, Hays AP, Rowland LP, Moghadaszadeh B, Beggs AH, Bhagat G. Adult-onset nemaline myopathy and monoclonal gammopathy. Arch Neurol. 2006;63:132-4.

17. Engel WK, Oberc MA. Abundant nuclear rods in adult-onset rod disease. J Neuropathol Exp Neurol. 1975;34:119-32.

18. Maeda MH, Ohta H, Izutsu K, Shimizu J, Uesaka Y. Sporadic late-onset nemaline myopathy as a rare cause of slowly progressive muscle weakness with young adult onset. Muscle Nerve. 2015;51:772-4.

19. Doppler K, Knop S, Einsele H, Sommer C, Wessig C. Sporadic late onset nemaline myopathy and immunoglobulin deposition disease. Muscle Nerve. 2013;48:983-8.

20. Desaegher J. Sporadic late onset nemaline myopathy with monoclonal gammopathy: hematopoietic stem cells therapy. Ann Phys Rehabil Med. 2015;58S:e145-52.

21. Novy J, Rosselet A, Spertini O, Lobrinus JA, Pabst T, Kuntzer T. Chemotherapy is successful in sporadic late onset nemaline myopathy (SLONM) with monoclonal gammopathy. Muscle Nerve. 2010;41:286-7.

22. Benveniste O, Laforet P, Dubourg O, Solly S, Musset L, Choquet $\mathrm{S}$, et al. Stem cell transplantation in a patient with late-onset nemaline myopathy and gammopathy. Neurology. 2008;71:531-2.

23. Belkhribchia MR, Tazi I, Louhab N, Kissani N, Mahmal L, Pereon Y. Autologous stem cell transplantationi in a patient with sporadic late-onset nemaline myopathy and monoclonal gammopathy: first Moroccan experience. Presse Med. 2017;46:122-5. 